\title{
A Few Bits Are Enough - ASIC Friendly Regular Expression Matching for High Speed Network Security Systems
}

\author{
Alex X. Liu Eric Norige \\ Dept. of Computer Science and Engineering \\ Michigan State University \\ East Lansing, MI, USA \\ \{alexliu, norigeer\}@cse.msu.edu
}

\author{
Sailesh Kumar \\ NetSpeed Systems \\ 2670 Seely Avenue, Building 11 \\ San Jose, CA 95134 \\ sailesh@netspeedsystems.com
}

\begin{abstract}
Regular Expression (RegEx) matching is the core operation of various network security devices such as IPSes. Despite much effort, it has remained an unsolved problem to achieve both high speed and low memory requirements.XFA, the state-of-the-art software RegEx matching solution, has two fundamental limitations: (1) XFA construction is hard to automate as it requires manual annotation by human experts, and (2) XFA is hard to implement in ASIC as the program executed upon reaching a state requires much of the complexity of a general purpose CPU. In this paper, we propose HASIC, a History-based Finite Automaton (HFA [11]) based RegEx matching scheme. HASIC can exponentially reduce state explosion by testing, setting, and clearing an auxiliary vector of history bits. Compared with XFA, HASIC advances the state of the art because it can be fully automated and it is ASIC friendly. HASIC only uses three simple bit operations and they are easy to implement in ASIC. We conducted experiments using real-world RegEx sets and various traffic traces. Experimental results show that for packet processing speed, software HFA runs an average of 3.34 times faster than XFA, for automata construction speed HFA is orders of magnitude faster than DFA, and for memory image size HFA is an average of 20 times smaller than DFA.
\end{abstract}

\section{INTRODUCTION}

\section{A. Motivation}

Deep Packet Inspection (DPI) is the core operation in a wide range of networking and security services (such as malware detection, data leak prevention, application protocol identification, load balancing, quality of service, differential billing, copyright enforcement, and usage monitoring) on most networking middleboxes and security devices (such as routers, firewalls, and Network Intrusion Detection/Prevention Systems (NIDS/NIPS)). In the past, string matching was used in DPI; nowadays, Regular Expression (RegEx) matching has become the industry standard because RegExes are more expressive than strings. Given a flow as a stream of bytes, a RegEx matching algorithm needs to determine which RegExes in a predefined set are matched in that flow. As each packet of each flow needs to go through RegEx matching, the memory and time efficiency of RegEx matching is critical to achieve low latency and high throughput. However, it is difficult to achieve both memory and time efficiency for RegEx matching. The Non-deterministic Finite Automata (NFA) model gives us

978-1-4799-1270-4/13/\$31.00 (c) 2013 IEEE the best memory efficiency as memory grows linear with the size of RegExes, but has the worst time efficiency because we need to maintain multiple active states and perform one memory lookup per active state for each input character. The Deterministic Finite Automata (DFA) model, on the other hand, gives us the best time efficiency of one lookup per character, but has the worst memory efficiency because of the well known state explosion problem - the number of DFA states can be exponential in the size of RegExes. Memory efficiency is critical for RegEx matching because it needs to run in SRAM to achieve high speed as SRAM is hundreds of times faster than DRAM.

To achieve high speed, RegEx matching algorithms need to be Application Specific Integrated Circuit (ASIC) friendly, i.e., they can be implemented in a small and fast ASIC block. The size of an ASIC block depends on the number of logic gates needed to implement the algorithm, and its speed (i.e., clock frequency) depends on circuit complexity. Not only chip fabrication cost is proportional to its die area, but also for networking and security devices such as IPSes, area efficiency of the circuit board is a critical issue.

\section{B. Limitations of Prior Art}

The software RegEx matching solution that represents the state of the art is XFA [20], [21]. An XFA is a DFA where each state is augmented with a program. The transition from a source state to a destination state triggers the execution of a program associated with the destination state. Despite its theoretical elegance, XFA has two fundamental limitations. (1) XFA does not have a fully automated construction algorithm (with a given RegEx set as input and a memory image for runtime packet processing as output) fundamentally because the XFA construction process requires human experts to annotate the given RegEx set [22]. Furthermore, both the nonautomated manual processing and the automated computation required in XFA construction may take a long time. For manual processing, XFA authors reported that the manual processing may take one hour even for one RegEx, and it is "prohibitively time-consuming" for some RegExes from Snort [20]. For automated computation, the required time to make the transitions deterministic varies for different RegEx sets, with $13 \%$ of the test set in [20] taking between 16 minutes 


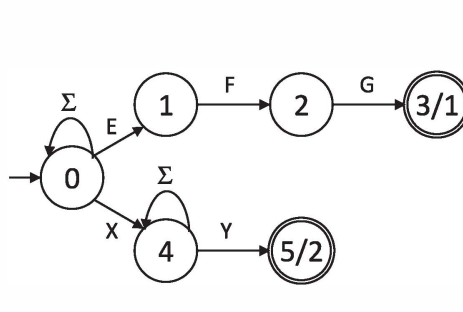

(a) NFA

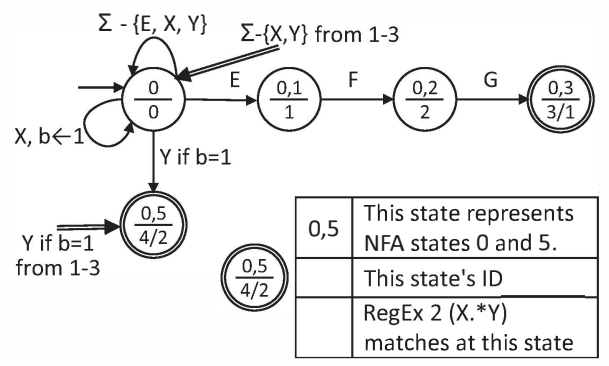

(b) HFA

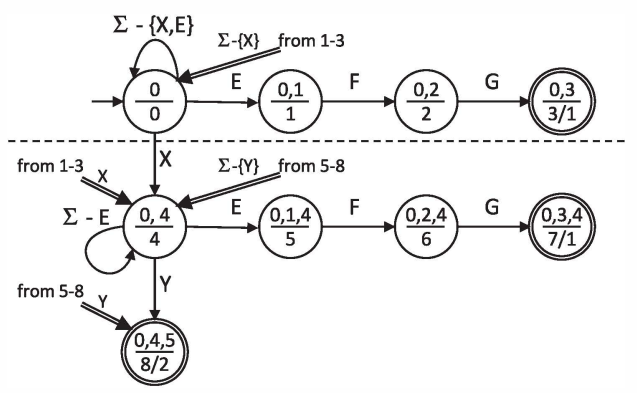

(c) DFA

Fig. 1. NFA, HFA, and DFA generated from RegEx set: $\{E F G, X . \star Y\}$

and 2 hours. Slow construction renders XFA unsuitable for applications where RegEx updating is frequent. (2) XFA is not ASIC friendly because the ASIC implementation of XFA would require much of the complexity of a general purpose CPU to implement the programs associated with states. Such an ASIC chip will not only be overly expensive but also too complex to develop and verify due to the complex interactions among different components. Furthermore, because each XFA state's program may take a very different amount of time to process, in the ASIC implementation of XFA, the processing elements at different stages would be very difficult to pipeline. These two reasons partially explain why XFA has not been implemented in ASIC since its invention. Nevertheless, XFA is no doubt an important RegEx matching solution as it shows the power of DFAs with annotated instructions.

\section{Proposed Approach}

In this paper, we propose HASIC, a History-based Finite Automaton (HFA) based RegEx matching scheme that is both fully automated and ASIC friendly. An HFA is a DFA with an auxiliary vector of bits (called history bits) where each transition is augmented with a boolean condition specified in history bits and some actions of setting/clearing history bits. The current HFA state, input character, and history bits jointly determine a single outgoing transition, and following this transition the actions to the history bits are applied.

We now explain why history bits can exponentially reduce the state explosion in DFAs. As a large number of RegExes can be partially matched in parallel, the DFA must track the progress of every combination of partial matches with a separate state. An exponentially large number of partial matches may occur leading to an exponential number of states. In real-life RegEx sets, many partially matched patterns are persistent; that is, once they have been found, they stay active for a long period of time, waiting to continue matching the rest of their pattern. Such partial matches are often the primary culprit behind the state explosion in DFAs. The bit-vector in HFAs can compactly represent these partial matches and therefore alleviate state explosion. For example, in the HFA in Figure 1(b) built from RegEx set $\{E F G, X . \star Y\}$, we use one bit $\mathrm{b}$ to remember the partial match of character $X$ for RegEx $X . \star Y$, which eliminates the duplication of the NFA states corresponding to RegEx EFG.

Although both HFA and XFA can exponentially reduce state explosion, they are fundamentally different. First, HFA places conditions and actions on transitions, using the history value to direct the operation of the automaton. XFA instead places actions on states and conditions on accepting states. This means that the sequence of states that XFA enters is not affected by its memory values, only whether or not it reports a match when it reaches an accept state. Second, they differ on operations. In HFA, the operations only include three simple bit operations: test, set, and clear, which are very easy to implement in ASIC. In XFA, the operations can be an arbitrary program, which requires CPU complexity to implement in ASIC. When limiting primitive XFA operations to a set of so called "efficiently implementable operations" [20], it is unclear what operations this set should include and whether some set of efficiently implementable operations can guarantee that an XFA can be constructed for any given RegEx.

Compared with XFA, HASIC advances the state of the art from two perspectives. First, HASIC construction can be fully automated (without human annotation). In this paper, we present efficient algorithms to construct an HFA from a RegEx set and then generate an optimized memory image for runtime packet processing. Second, HASIC is ASIC friendly. HASIC only involves three simple bit operations: test, set, and clear, and only adds a small fixed-size bit array to the state of each flow, which makes storing and restoring the active state of each flow efficient. Furthermore, these operations are combined without the need for a software layer to tie them together, allowing us to represent the processing logic with a simple fixed combination of gates.

\section{Challenges and Proposed Solutions}

There are two key challenges in HASIC: (1) automated and fast HFA construction (converting a RegEx set to an HFA), (2) memory image construction (converting an HFA to a memory image) for fast packet processing.

Automated and Fast HFA Construction: Automatic HFA construction is challenging because of two reasons. First, the number of choices for history bits is exponential in the number of NFA states; yet, choosing the right set of history bits is most critical for eliminating state explosion. Second, the number of history bits in a high speed implementation is limited; we must construct an automaton that uses no more than this many history bits. We solve this by identifying NFA states that stay active over many transitions, and ranking them so that we can choose the best states to use. Fast HFA construction is challenging because the intermediate DFA is often exponential (in the number of NFA states) to construct in both time and space; and sometimes the DFA is too big 
to be practically built. In this paper, we propose a fast and automated HFA construction algorithm whose time and space complexity is linear in the size of the final HFA, which is the best possible complexity for HFA construction. Our key idea is to eliminate the bit states from the NFA before doing subset construction. We can then modify the generated states to compensate, allowing us to avoid exploring all the potential DFA states.

Memory Image Construction for Fast Packet Processing: The number of memory reads per input character for finding the next state(s) is critical for RegEx algorithms to achieve high throughput. It is challenging to minimize the number of memory reads per character in an HFA because there can be multiple HFA transitions for a state and character pair. In this paper, we minimize the number of transitions to be searched by merging compatible transitions into a single transition with the same net effect. To further reduce the size of each transition in memory, we compress the actions by using an auxiliary action mask table to store atomic actions so that the actions for any transition can be represented as the union of these atomic actions; thus, for each transition, instead of storing its actions, we only need to store the indexes of the atomic actions in the action mask table.

\section{E. Key Novelty and Contributions}

The key novelty is in proposing automatic HFA construction algorithms and memory image construction algorithms, where we identify many optimization opportunities. The main contribution is in proposing an automated and ASIC friendly RegEx matching scheme. Specifically, we make the following key contributions. First, we propose an automated and optimized HFA construction algorithm, and then propose a fast HFA construction algorithm with linear complexity in the final HFA size. Second, we propose an optimized memory image construction algorithm for fast runtime packet processing. Finally, we conducted experiments using real-world RegEx sets and various traffic traces. As we cannot construct XFA from our RegEx sets, we estimate the packet processing speed on our hardware based on the results reported in [20]. Our results show that HFA runs an average of 3.34 times faster than XFA. In comparison with DFA, for automata construction speed, HFA is orders of magnitude faster than DFA; for memory image size, HFA is an average of 20.4 and 16.5 times smaller than DFA for memory bus width of 16 and 32 bytes, respectively.

\section{RELATED WORK}

Existing RegEx matching schemes fall into three categories based on their underlying implementation technology: software based, FPGA based, and TCAM based.

Software-based schemes are generally based on deterministic automata to achieve high throughput. The difference between them is in their approach to solving the DFA state explosion problem. We divide software-based schemes based on whether they introduce auxiliary memory to the automaton or not. Schemes that do not introduce auxiliary memory include $\mathrm{D}^{2} \mathrm{FA}$ [12], mDFA [24], and Hybrid-FA [4]. D ${ }^{2} \mathrm{FA}$ achieves significant compression of the transition table, but does not solve the exponential explosion in the number of states. mDFA and Hybrid-FA avoid building too large a DFA by either building multiple DFA or by producing a partiallydeterministic automaton. In both cases, there will be multiple simultaneous active states, causing a large reduction in throughput. Schemes that augment auxiliary memory include XFA [20], [21], extended-FA [6], and HFA [11]. XFA and extended-FA both propose hardware designs that are, in broad strokes, a plain DFA that processes all traffic plus a much more complex logic that handles the parts of the RegExes that are too complex to add to the DFA. The complexity of this second layer makes them unsuitable for ASIC implementation. Additionally, the task of coupling these two layers together to achieve guaranteed high performance is even more difficult, as the second layer's processing cost per input byte is highly variable. In [11], Kumar et al. briefly proposed the theoretical model of HFA and a manual HFA construction method that requires human experts to design history bits. However, they proposed neither the methods for the automatic construction of HFAs nor the methods for generating memory images for runtime packet processing. Furthermore, the manual HFA construction method in [11] requires first constructing a DFA from the given RegEx set and then constructing the HFA from the DFA. Although the final HFA is memory efficient, the intermediate DFA is often exponential (in the number of NFA states) to construct in both time and space; and sometimes the DFA is too big to be practically built. Note that the solution for handling large counters in [11] can be applied in our scheme as well.

The field of regular expression matching using FPGA includes a huge breadth of work [3], [8]-[10], [13], [15], [19], [23]. These techniques all develop a circuit on FPGA that takes in packet data and reports match information. Important to their methods is using the reprogrammability of the FPGA to have the flexibility to handle many pattern sets. These techniques are effective for a fixed pattern set or for environments where the pattern matching tool can be taken offline without penalty. Because the re-synthesis procedure to update the patterns is complex and requires taking the FPGA offline, FPGA solutions have issues with practical deployment in many scenarios. As well, the matching state of many FPGA solutions is large, making it expensive to save and restore this state when matching a large number of interleaved network flows. As an example, in Bando et al. [3], the internal state includes 1.5 Kbits of status flags for string matching modules. This makes the handling of interleaved flows much more complex, as saving and loading that state is very expensive.

A newer line of research is the use of TCAM technology to encode pattern matching automata [14], [17]. TCAM are content addressable memory with wild-cards, meaning that a binary string is input, and the set of ternary patterns stored in the memory are checked to find the first pattern that matches the query. An SRAM associated with the TCAM allows a value to be associated with each pattern in the TCAM. The transition table of an automaton can be implemented by creating query strings that indicate the current state and input character and storing the destination state of the transition in the SRAM. The downside of TCAM is its high power usage, as every query is matched against every pattern. 


\section{AUtOMATic HFA CONSTRUCTION}

\section{A. Basic Construction Method}

The original algorithm to construct an HFA from a RegEx set first constructs an NFA and uses subset construction to create the corresponding DFA. The label of each DFA state is the subset of NFA states that the DFA state is constructed from. Second, the DFA is "folded" into an HFA by repeatedly turning a single NFA state into a history bit, removing this label from all DFA states, and merging those DFA states that now have identical NFA state labels. We call the removed NFA states "bit states". To convert an NFA state $s$ into a bit state, we partition the DFA into two groups: $P$, which consists of the states that have the NFA state $s$ in their label, and $N$, which consists of those that do not. For example, to convert NFA state 4 in Figure 1(a) into a bit state, we partition the DFA in Figure 1(c) into two groups: $P=\{4,5,6,7,8\}$ and $N=\{0,1,2,3\}$, those states that have the NFA state 4 and those that do not. We use an example state label $\frac{0,3,4}{7 / 1}$ to explain the way that we label DFA (or HFA) state in this paper: $0,3,4$ is the set of NFA states that corresponds to this DFA state, 7 is the DFA state ID, and $/ 1$ denotes that this is an accepting state and the RegEx ID 1 (namely EFG) is matched upon reaching this state. Removing label $s$ from each state in $P$ will allow us to merge each state and their transitions in group $P$ with the corresponding state and transitions in $N$. For example, removing NFA state label 4 from each DFA state in $P=\{4,5,6,7,8\}$ will allow us to pairwise merge DFA states 4 and 0,5 and 1, 6 and 2, and 7 and 3.

Transitions leaving a state in $P$ can now be taken when the corresponding history bit for $N$ is set and transitions leaving a state in $N$ can only be taken when that bit is clear. For example, in Figure 1(b), the transitions from HFA states 1, 2, and 3 to state 4 on character $Y$ can only be taken when $b$ is set. The transitions that were going from $P$ to $N$ must clear the history bit, and from $N$ to $P$ must set it. For example, in Figure 1(b), the transition from HFA state 0 to 0 on character $\mathrm{X}$ corresponds to the transition from DFA state 0 to 4 . Because this transition goes from $N$ to $P$, it sets bit $b$. Transitions that stay within a group do not need an action to modify the history bits. For example, in Figure 1(b), in the transitions among HFA states $0,1,2$, and 3 , there is no action to modify bit $b$. Repeating the above process constructs an HFA with multiple history bits.

\section{B. Bit State Selection}

Choosing bit states is critical for HFA. The best case for compressing the DFA by converting an NFA state $s$ into a history bit is when the DFA states that include $s$ in their labels exactly mirror the DFA states that do not, which allows us to halve the number of automaton states. For example, in Figure I, by choosing NFA state 4 to be a bit state, we almost halve the number of HFA states.

Before presenting our method for choosing the right bit states, we first introduce some new concepts. The self-looping degree of an NFA state is defined as the percentage of the number of input characters that the state transitions to itself on. An NFA state is complete-self-looping if its selflooping degree is $100 \%$. For example, in Figure 1(a), both states 0 and 4 are complete-self-looping. RegExes with .* cause most complete-self-looping states. Once a complete-selflooping becomes active, it remains active. An NFA state $s_{1}$ shadows another state $s_{2}$ if and only if every time when state $s_{2}$ is active, $s_{1}$ is also active. For example, in Figure 1(a), state 0 shadows every other state, and state 4 shadows state 5 .

The shadowing relationship reduces state explosion by eliminating the combinations of states for which we need to generate new DFA states. For example, in Figure 1(a), we do not need to generate a new DFA state for the combination of states $\{0,5\}$ because whenever state 5 is active, state 4 is active. Two NFA states $s_{1}$ and $s_{2}$ are exclusive if and only if they cannot be simultaneously active. For example, in Figure 1(a), states 1, 2, and 3 are mutually exclusive. Exclusive relationship also reduces state explosion by eliminating the combinations of states that we need to generate new DFA states for. For example, in Figure 1(a), we do not need to generate a new DFA state for the combination of states $\{1,2\}$ because they cannot be simultaneously active. NFA states $s_{1}$ and $s_{2}$ are independent if and only if two conditions are satisfied: (1) there is no shadowing relationship between them, and (2) they are not exclusive.

Independent states cause state explosion in DFAs. Given an NFA with $n$ independent persistent states and $m$ other states, using $d(m)$ to denote the size of the DFA constructed only from the $m$ states, the size of the final DFA is in the order of $2^{n} * d(m)$. This comes from the $d(m)$ states being copied for all $2^{n}$ ways for the independent states to be active.

For an NFA state that is independent from most of other states, if we choose it to be a bit state, then we almost halve the DFA size. As NFA states with a high self-looping degree tend to remain active for a long time, we use the NFA states with a high self-looping degree that are shadowed by complete-selflooping states as bit states, as these are likely to be independent with a large number of other states.

\section{HFA Construction without DFA}

In constructing an HFA from a RegEx set, the intermediate DFA may be too big to be practically generated due to state explosion, even if the final HFA is small. Next, we present our HFA construction algorithm that can directly build the HFA from an NFA without generating and storing the intermediate DFA. Before we present our algorithm, we first introduce a new concept of equivalent state classes: given an NFA with a set of bit states $B$, for two DFA states that correspond to two sets of NFA states $S_{1}$ and $S_{2}$, if the two NFA state sets only differ on bit states (i.e., $S_{1}-B=S_{2}-B$ ), then the two DFA states are equivalent with respect to bit state set $B$. This relationship partitions the DFA states into equivalence classes. Considering the example in Figure 1, when choosing NFA state 4 as a bit state, the DFA states 1 and 5 are in the same equivalence class as they correspond to NFA state sets $\{0,1\}$ and $\{0,1,4\}$, respectively.

The this HFA construction algorithm is similar to the standard subset construction algorithm as in DFA construction, but it only generates one HFA state per equivalence class. Let $B$ be the set of bit states. Each time we generate a DFA state that corresponds to a set of NFA states $S$, we append its transitions to the HFA state $S-B$. For each transition from DFA state $S$ to DFA state $D$, we add an HFA transition, 
with a condition and an action, from $S-B$ to $D-B$ on the same character. The condition corresponds to $S \cap B$, meaning that this transition can only be taken when the history bits corresponding to $S \cap B$ are set and the remaining history bits are clear. The action consists of two parts: setting the history bits corresponding to $(D-S) \cap B$ and clearing the history bits corresponding to $(S-D) \cap B$.

An HFA with a vector $H$ of $k$ history bits, has 5-tuple transitions $(S, c, \mathbb{C}, \mathbb{A}, D)$ which are the source state, input character, condition, action, and destination state. The source state and destination state of an HFA are HFA states, which are written as a set of NFA states. The condition of an HFA transition is represented as a vector $\mathbb{C}$ of $k$ ternary bits, denoting the condition $\wedge_{i=0}^{k-1}(\mathbb{C}[i]=H[i])$. A ternary bit has three possible values: 0,1 , or *. Note that for each $0 \leq i \leq k-1$, when $\mathbb{C}[i]$ is $*, \mathbb{C}[i]=H[i]$ is true regardless of the value of $H[i]$. The action of an HFA transition is represented as a vector $\mathbb{A}$ of $k$ bit-wise operations. Each bitwise operation is either set (denoted as $s$ ), clear (denoted as $\mathrm{c}$ ), or do-nothing (denoted as $\mathrm{n}$ ). For each $0 \leq i \leq k-1$, $\mathbb{A}[i]=\mathrm{s}$ means the action assigns 1 (sets) to $H[i], \mathbb{A}[i]=\mathrm{c}$ means the action assigns 0 (clears) to $H[i]$, and $\mathbb{A}[i]=\mathrm{n}$ means the action does nothing to $H[i]$. Table I shows example transitions with 3 history bits for a HFA state and character pair. The pseudocode of this HFA construction algorithm, called $\mathrm{HASIC}_{D}$ is shown in the appendix.

\begin{tabular}{|l|l|l|}
\hline Condition & Action & Destination State \\
\hline$* 00$ & $\mathrm{nnn}$ & $\{1\}$ \\
$* 10$ & $\mathrm{n} \mathrm{n}$ & $\{1\}$ \\
$* 01$ & $\mathrm{nnn}$ & $\{1,5\}$ \\
$* 11$ & $\mathrm{n} \mathrm{n}$ & $\{1,5\}$ \\
\hline
\end{tabular}

TABLE I. EXAMPLE TRANSITIONS BEFORE OPTIMIZATION

\section{Transition Table Optimization}

The above HFA construction algorithm avoids DFA state explosion by merging many variants of the same NFA states into one HFA state; however, all it adds each DFA transition to the HFA. Next, we introduce our HFA transition table optimization algorithm that can efficiently store transitions while allowing fast transition lookup. We first introduce a new concept called mergeable bit actions. Given an action $a$ on a ternary bit $t$, we use $a(t)$ to denote the resulting value after applying action $a$ on $t$. Two action and ternary bit pairs $\left(a_{1}, t_{1}\right)$ and $\left(a_{2}, t_{2}\right)$ are mergeable if and only if there exists an action $a_{3}$ so that $a_{1}\left(t_{1}\right)=a_{3}\left(t_{1}\right)$ and $a_{2}\left(t_{2}\right)=a_{3}\left(t_{2}\right)$. We call $a_{3}$ a merged action of $\left(a_{1}, t_{1}\right)$ and $\left(a_{2}, t_{2}\right)$. For example, $(n, 0)$ and $(\mathrm{s}, 1)$ are mergeable and the merged action is $n$. One merging may have two merged actions, either $n$ and $c$ or $n$ and $s$. For example, action $\mathrm{n}$ on bit 0 and action $\mathrm{c}$ on bit 0 have two possible merged actions - $\mathrm{n}$ and $\mathrm{c}$. In such cases, we choose $n$ to be the merged action because it has less bit operations than $\mathrm{c}$ or $\mathrm{s}$. Note that the choice of merged actions can never be between $c$ and $s$ because their results are always different. Table II shows the merged actions of all possible pairs of bitaction pair, where - denotes that the two bit-action pairs are not mergeable.

Now we introduce another concept called mergeable transitions and discuss how to minimize HFA transitions by

\begin{tabular}{|c|c|c|c|c|c|c|c|c|}
\hline n0 & $\mathrm{n} 1$ & $\mathrm{n} *$ & $\mathrm{CO}$ & $\mathrm{C} 1$ & $C^{*}$ & s0 & $\mathrm{s} 1$ & $\mathrm{~S} *$ \\
\hline n0 & $\mathrm{n}$ & $\mathrm{n}$ & $\mathrm{n}$ & $\mathrm{C}$ & $\mathrm{C}$ & - & $\mathrm{n}$ & - \\
\hline $\mathrm{n}$ & $\mathrm{n}$ & $\mathrm{n}$ & $\mathrm{n}$ & - & - & $\mathrm{S}$ & $\mathrm{n}$ & $\mathrm{S}$ \\
\hline n* & $\mathrm{n}$ & $\mathrm{n}$ & $\mathrm{n}$ & - & - & - & $\mathrm{n}$ & - \\
\hline $\mathrm{n}$ & $\mathrm{n}$ & $\mathrm{n}$ & $\mathrm{n}$ & $\mathrm{C}$ & $\mathrm{C}$ & - & $\mathrm{n}$ & - \\
\hline C 1 & - & - & C & C & C & - & - & - \\
\hline$C^{*}$ & - & - & $\mathrm{C}$ & C & C & - & - & - \\
\hline s0 & $S$ & - & - & - & - & $S$ & $\mathrm{~S}$ & $\mathrm{~S}$ \\
\hline$n$ & $\mathrm{n}$ & $\mathrm{n}$ & $\mathrm{n}$ & - & - & $\mathrm{S}$ & $\mathrm{n}$ & $\mathrm{S}$ \\
\hline S* & $S$ & - & - & - & - & $S$ & $\mathrm{~S}$ & $S$ \\
\hline
\end{tabular}

TABLE II. HFA TRANSITION MERGEABILITY TABLE

identifying and merging such transitions. In an HFA with $k$ history bits, a transition $\left(S, c, \mathbb{C}_{1}, \mathbb{A}_{1}, D\right)$ and a transition $\left(S, c, \mathbb{C}_{2}, \mathbb{A}_{2}, D\right)$ are mergeable if and only if both of the following conditions are satisfied: (1) $\mathbb{C}_{1}$ and $\mathbb{C}_{2}$ differ in only one bit and (2) for $0 \leq i \leq k-1$, $\left(\mathbb{A}_{1}[i], \mathbb{C}_{1}[i]\right)$ and $\left(\mathbb{A}_{2}[i], \mathbb{C}_{2}[i]\right)$ are mergeable. For these two mergeable transitions, assuming $\mathbb{C}_{1}$ and $\mathbb{C}_{2}$ differ in bit $i$, we merge them into one rule by both replacing $\mathbb{C}_{1}[i]$ by $*$ and replacing $\mathbb{A}_{1}[i]$ by the merged action from Table II. For example, in Table I, the first two transitions and the last two transitions are mergeable. Table III shows the two merged actions. This optimization allows many HFA transitions to be stored in a small memory while allowing fast packet processing.

\begin{tabular}{|l|l|l|}
\hline Condition & Action & Destination State \\
\hline$* * 0$ & $\mathrm{n} \subset \mathrm{n}$ & $\{1\}$ \\
$* * 1$ & $\mathrm{n} \mathrm{n}$ & $\{1,5\}$ \\
\hline
\end{tabular}

TABLE III. TABLE I TRANSITIONS AFTER OPTIMIZATION

\section{FAST HFA CONSTRUCTION}

In this section, we propose the first algorithm to generate deterministic automata without exploring all the possible NFA state interactions. Compared with the HFA construction algorithm in Section III, this algorithm runs significantly faster at the price of producing a possibly larger HFA.

\section{A. Observation and Basic Ideas}

In observing the HFAs that our previous algorithm constructed from real-life RegEx sets, we observe that many HFA states have exactly $2^{n}$ outgoing transitions for a particular input character where $n$ is the number of bit states. This occurs when $n$ bit states have departing transitions to distinct NFA states on the same input character. When we merge all the transitions from all the DFA states in an equivalence class, our previous algorithm has to create a conditional transition for each reachable combination of history bits. This constitutes a significant portion of the HFA construction time. To speed up HFA construction, our key idea is to simply assume each bit state is independent from all other states; thus, we can precompute an incoming transition table and an outgoing transition table, which we call the mixin incoming table and mixin outgoing table, respectively. These two tables consist of all the transitions introduced by all combinations of bit states. Then, we can mix this table with the transition table of each HFA state. This may introduce some transitions that can 
never be taken, but they do not affect the correct execution of the automaton. We choose the term "mixin" because of its resemblance of the use of mixin classes in object-oriented programming, where a mixin is a class that provides a certain functionality to be inherited or reused by derived classes.

To construct an HFA from an NFA using this method, we first identify bit states as described earlier. Second, we generate a mixin incoming table that is constructed from all incoming transitions to bit states and a mixin outgoing table that is constructed from all outgoing transitions from bit states. Third, we remove all bit states and their incoming and outgoing transitions from the NFA to produce a pruned NFA that has only non-bit states. Finally, we generate HFA states using subset construction on the pruned NFA, "mixing in" the transition information from the two mixin tables.

To illustrate this process, we will show step by step conversion of the example NFA in Figure IV-A into the HFA in Figure IV-D.

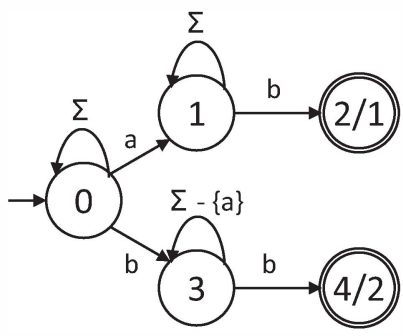

Fig. 2. Input NFA

\section{B. Bit State Pruning}

Given an NFA and its bit states, we produce a pruned NFA by removing the bit states from the NFA as well as all their incoming and outgoing transitions. The information about these bit states, which is missing in the pruned NFA, will be kept in two mixin tables. When a history bit is set, this impacts the action of a transition and/or its destination. The mixin tables capture both of these effects and allow us to apply them to the full HFA. In the NFA in Figure IV-A, which is constructed from the regular expressions $\mathrm{a}_{*} \star \mathrm{b}$ and $\mathrm{b}$ [ $\left.{ }^{\wedge} \mathrm{a}\right] * \mathrm{~b}$, we choose states 1 and 3 as the bit states, which means that we have two history bits that correspond to NFA states 1 and 3. The pruned HFA is shown in Figure IV-B.

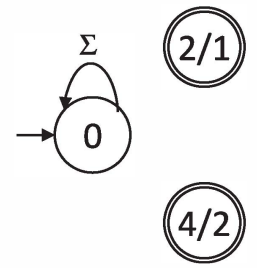

Fig. 3. Pruned NFA

\section{Mixin Table Generation}

We use two tables to store the information about bit states: the mixin incoming table and the mixin outgoing table. For the mixin incoming table, we generate it directly from the NFA. For the mixin outgoing table, we first generate an outgoing table for each bit state and then merge them into one table. Note that these two tables are only used for HFA construction and they are not part of the final HFA memory image.

To generate the mixin incoming table, we simply record all the transitions from non-bit states to bit states. Figure IV-C shows the mixin incoming table for the NFA in Figure IV-A, whose entries are three tuples, $(q, c, \mathbb{A})$. Note that the source field of this table is a single NFA state. The first entry shown comes from the incoming transition to NFA state 1 on input character a. The source field of the transition is 0 , indicating that this is only available from HFA states constructed from NFA state 0 . The action " $s n$ " means that we should set the first history bit and do nothing to the second history bit. Similarly, on b, the action "ns" sets the second history bit.

\begin{tabular}{|c|c|c|}
\hline Src. & input & Act. \\
\hline 0 & $\mathrm{a}$ & $\mathrm{sn}$ \\
& $\mathrm{b}$ & $\mathrm{ns}$ \\
\hline
\end{tabular}

TABLE IV. MIXIN INCOMING TABLE

The outgoing table for a bit state has entries that are 4tuples, $(c, b, \mathbb{A}, D)$, representing an input character, single history bit, action, and destination state. The input character and history bit value uniquely determine an action and a destination state. For input characters that are not shown, the default entry is an action that does nothing and an empty destination state, marked "else". To generate the mixin outgoing table entries for a bit state, we first examine its outgoing transitions that are not looping back to itself. Figure IV-C shows the mixin outgoing table of bit state 1 , which has a transition on $b$ to state 2. The corresponding entry in this table means that in the final HFA, whenever bit state 1 is active, on character b, we take no additional action and we transition to a combination of NFA states containing state 2 . For transitions that leave a bit state to go to another bit state, the outgoing table entry sets the history bit for the destination state instead of putting that state in the destination set. When processing an input character for which some bit state has a self-looping transition, the corresponding history bit will not change. But we must clear the history bit upon processing a character lacking a self-loop transition. Figure IV-C shows an example of this: bit state 3 does not have a transition to itself on character a, thus we need the first entry in its outgoing table, which clears the history bit for state 3 when it is set.

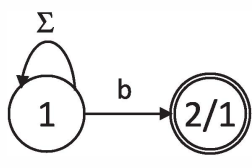

\begin{tabular}{|c|c|c|c|}
\hline input & Cond. & Act. & Dest. \\
\hline$b$ & $\begin{array}{l}1 \\
0\end{array}$ & $\begin{array}{l}\mathrm{nn} \\
\mathrm{nn}\end{array}$ & $\begin{array}{c}\{2\} \\
\emptyset\end{array}$ \\
\hline$*$ & $*$ & $\mathrm{nn}$ & $\emptyset$ \\
\hline
\end{tabular}

Table V. Bit State 1 Outgoing Table

After we generate a mixin outgoing table for each bit state, we merge these individual outgoing tables into a single mixin outgoing table, which will be used during the subset construction process. The entries in this table are 4-tuples, $(c, \mathbb{C}, \mathbb{A}, D)$, similar to the outgoing table for a single state, but with $k$-bit conditions. Given multiple individual mixin outgoing tables, we construct all combinations of rules in the 


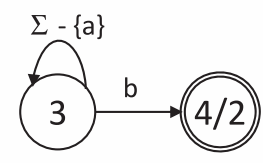

else:

\begin{tabular}{|c|c|c|c|}
\hline input & Cond. & Act. & Dest. \\
\hline \multirow{2}{*}{$\mathrm{a}$} & 1 & $\mathrm{nc}$ & $\emptyset$ \\
& 0 & $\mathrm{nn}$ & $\emptyset$ \\
\hline \multirow{2}{*}{$\mathrm{b}$} & 1 & $\mathrm{nn}$ & $\{4\}$ \\
& 0 & $\mathrm{nn}$ & $\emptyset$ \\
\hline \hline$*$ & $* *$ & $\mathrm{nn}$ & $\emptyset$ \\
\hline
\end{tabular}

TABLE VI.

Bit State 3 Outgoing Table

input tables that have the same input character, $\sigma$. The resulting destination of two rules is the union of their destination state sets. The rule for merging actions is based on NFA semantics, where a state becoming active takes precedence over that state becoming inactive from the lack of a transition. Thus, the result of merging two actions is $s$ if either input is $s, c$ if some action is $c$, otherwise $n$. For example, the first entry in Figure IV-C is the result of merging the entry for a in Figure IV-C with the default entry from Figure IV-C. For actions, we have $n n+n c=$ $\mathrm{nc}$ as the merging result. For destinations, we have $\emptyset \cup \emptyset=\emptyset$ as the merging result. Since we have two entries on b in each table, the mixin outgoing table will have 4 entries, as shown in Figure IV-C.

\begin{tabular}{|c|c|c|c|}
\hline input & Cond. & Act. & Dest. \\
\hline \multirow{2}{*}{$\mathrm{a}$} & *1 & $\mathrm{nc}$ & $\theta$ \\
\hline & $* 0$ & $\mathrm{nn}$ & $\emptyset$ \\
\hline \multirow{4}{*}{ b } & 00 & $\mathrm{nn}$ & $\theta$ \\
\hline & 10 & $\mathrm{nn}$ & $\{2\}$ \\
\hline & 01 & $\mathrm{nn}$ & $\{4\}$ \\
\hline & 11 & $\mathrm{nn}$ & $\{2,4\}$ \\
\hline * & ** & $\overline{n n}$ & $\theta$ \\
\hline
\end{tabular}

Fig. 4. Mixin Outgoing Table for $1 \& 3$

\section{HFA Transition Table Generation}

We now construct HFA from the pruned NFA, not the original NFA. We still use subset construction considering all possible combination of states in the pruned NFA. But note that the number of all combinations of pruned NFA states is orders of magnitude smaller than that of the original NFA. This explains why this HFA construction method is much faster than the one in Section III. In generating transitions, we mix in the transitions in the mixin transition table as follows. The transitions generated from the pruned NFA, which has no bit states, can be represented as 3-tuples: source NFA state set, input character, and destination NFA state set. Let $(S, c, D)$ denote a transition generated from the pruned NFA. The transitions in the mixin incoming table can be represented as 3-tuples: source NFA state, input character, and action. Let $\left(q_{i}, c_{i}, \mathbb{A}_{i}\right)(1 \leq i \leq m)$ denote the $m$ entries of the mixin incoming table with $c_{i}=c$ and $q_{i} \in S$. We will merge all these actions into the result, so we can write $M$ as the result of merging all the $\mathbb{A}_{i}$. Recall that the result of merging two actions is $\mathrm{s}$ if either input is $\mathrm{s}, \mathrm{c}$ if some action is $\mathrm{c}$, otherwise n. Let $\left(c_{j}, \mathbb{C}_{j}, \mathbb{A}_{j}, D_{j}\right)(1 \leq i \leq n)$ denote the $n$ entries of the mixin outgoing table with $c_{j}=c$. In generating the transitions for each NFA state, we merge the destination set constructed from the pruned NFA with the two mixin tables in a manner similar to how we merge individual mixin outgoing tables. For each entry $\left(c_{j}, \mathbb{C}_{j}, \mathbb{A}_{j}, D_{j}\right)$ in the mixin outgoing table, we create an HFA transition $\left(S, c, C_{j}, M+\mathbb{A}_{j}, D \cup D_{j}\right)$. In our example, the subset construction process generates an HFA state for NFA state $\{0\}$ and constructs the transition $(\{0\}, a,\{0\})$. This is augmented by the mixin tables to become $(\{0\}, \mathrm{a}, * 1, \mathrm{sc},\{0\})$ and $(\{0\}, \mathrm{a}, * 0, \mathrm{sn},\{0\})$, which can be compressed into the transition from HFA state 0 to itself on input character a, setting the history bit for bit state 1 and clearing the history bit for bit state 3 . The subset construction process also constructs a similar transition for b: $(\{0\}, \mathrm{b},\{0\})$. This transition is expanded to four transitions, each with different destinations: ( $\{0\}, \mathrm{b}, 00, \mathrm{nn},\{0\}),(\{0\}, \mathrm{b}, 10, \mathrm{nn},\{0,2\})$, $(\{0\}, b, 01, n n,\{0,4\}),(\{0\}, b, 11, n n,\{0,2,4\})$. The newly reachable states from $\{0\}$ have their transition tables generated in the same manner until all states are constructed. Applying this algorithm to the pruned NFA in Figure IV-B, the Mixin Incoming Table in Figure IV-C and the Mixin Outgoing Table in Figure IV-C produces the HFA in Figure IV-D.

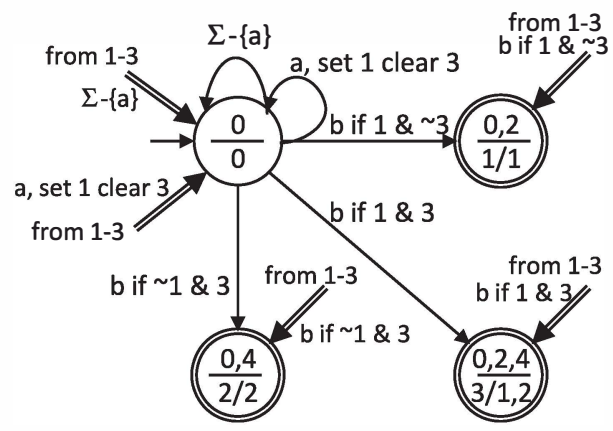

Fig. 5. Output HFA

The work necessary to produce HFA by this process is not proportional to the size of the DFA. When we prune the bit states that cause exponential increase in DFA size and construct HFA using mixin tables, we avoid enumerating exponentially many combinations of NFA states. Our experimental results show that this fast HFA construction algorithm produces HFAs of similar size as our previous HFA construction algorithm at orders of magnitude higher speed.

\section{EXPERIMENTAL RESULTS}

We demonstrate the capability of HASIC by comparing it with DFA for speed because DFA is the fastest (although the biggest), with NFA for memory size because NFA is the smallest (although the slowest), and with XFA because XFA represents the state-of-the-art. We compare the construction time of the direct HFA construction algorithm in Section III-C, denoted $\mathrm{HASIC}_{D}$, and the mixin-based HFA construction algorithm in Section IV, denoted $\mathrm{HASIC}_{M}$, with that of DFA and NFA. We cannot compare with XFA construction time as it cannot be automated and XFA construction code is not available.

\section{A. Data Set}

The RegEx sets that we use come from a variety of sources. Sets C7, C8, and C10 are proprietary and come from a large networking vendor. Sets S24, S31, S34, and B217 are public sets from Snort [18] and Bro [16] that has been used in prior literature [5]. Within C7, S31, and B217, there are a number of RegExes with . $\star s$ that have been commented off. We further 
created RegEx sets C7p, S31p, and B217p by restoring the RegExes containing . $\star \mathrm{S}$ from these three sets respectively. As $\mathrm{C} 7 \subset \mathrm{C} 7 \mathrm{p}, \mathrm{S} 31 \subset \mathrm{S} 31 \mathrm{p}, \mathrm{B} 217 \subset \mathrm{B} 217 \mathrm{p}$, we focus the collection of RegEx sets C7p, C8, C10, S24, S31p, S34, and B217p, denoted as BCS.

The 217 RegExes in Bro217 are almost entirely string matching, although the additional RegExes that we restored have one to three wildcard closures $(. \star)$ each. The Snort sets have a higher density of wildcard and near-wildcard (e.g., [ $\backslash r \backslash n] *$ ) closures, and the C7, C8, C10 sets have a very high density of wildcard and wildcard closures, with as many or more closures than the number of RegExes. The density of these closures makes their corresponding DFA much larger. To determine the scaling properties of HASIC, we created an additional set Scale by combining all the distinct RegExes from S24, S31p, and S34. Table VII summarizes the properties of these RegEx sets with their corresponding numbers of NFA states, DFA states, HFA states (by $\mathrm{HASIC}_{M}$ ), and history bits. Note that the HFAs generated by $\mathrm{HASIC}_{M}$ and $\mathrm{HASIC}_{D}$ for the same RegEx set have almost the same number of states.

\begin{tabular}{|r|r|r|r|r|r|}
\hline Set & RegExes & NFA St. & DFA St. & HASIC $_{M}$ St. & Hist. Bits \\
\hline B217p & 224 & 2553 & - & 16527 & 10 \\
\hline C7p & 11 & 295 & 244366 & 616 & 12 \\
\hline C8 & 8 & 99 & 3786 & 117 & 7 \\
\hline C10 & 10 & 123 & 19508 & 238 & 9 \\
\hline S24 & 24 & 702 & 10257 & 925 & 7 \\
\hline S31p & 40 & 1436 & 39977 & 2323 & 18 \\
\hline S34 & 34 & 1003 & 12486 & 1362 & 8 \\
\hline Scale & 77 & 2631 & 593810 & 7401 & 30 \\
\hline
\end{tabular}

TABLE VII. RegEx Set Properties

We evaluated our solution using both synthetic and reallife traffic traces. The synthetic trace comes from Becchi et al.'s flow generator [7], which is a useful tool for generating synthetic streams of various degrees of maliciousness based on given RegExes. The degree of maliciousness depends on parameter $p_{M}$ where a higher value indicates more malicious traffic. More specifically, the trace is generated such that with probability $p_{M}$, each input character transitions the automaton away from the start state, which will cause a large amount of the DFA states to be visited, forcing a small cache hit rate and thus many accesses to main memory. Our synthetic traces are generated with the default $p_{M}$ values of $0.35,0.55,0.75$, 0.95 as specified in the tool. Furthermore, we generate a trace called rand consisting of purely random data.

To test the overhead of handling interleaved packets from many simultaneous flows, we also use real traffic traces. This allows us to test not only raw processing speed but also the ability to save and load the matching state for each flow. We use three sources for realistic trace data: (1) the DARPA intrusion detection data set, generated by the MIT Lincoln Laboratory (LL) [1], (2) traces captured during the 2009 InterService Academy Cyber Defense Competition (CDX) [2], and (3) a locally gathered traffic trace of a student lab PC (RL). For LL, we process the ten traces from week 5 for a total of 5.8GB. For CDX, we process the Border Data Capture traces 3 through 8 , for a total of 550M. For RL, we capture 10 traces, the size of each is $0.1 \mathrm{~GB}$.

\section{B. Metrics \& Experimental Setup}

To compare these algorithms, we measure automaton construction time, memory image size, and packet processing throughput. Memory image size is measured by the amount of contiguous memory needed to store an automaton. Throughput is measured by the RegEx matching time per byte in processing packets. Error bars in graphs represent standard deviation. The experiments are carried out on a server with a Sandy Bridge Core(I7-2600K@3.4GHz) and 8GB RAM. The image construction code is $1.5 \mathrm{~K}$ lines of OCaml, and the pattern matching engine is 300 lines of $\mathrm{C}++$. For all experiments, we use a single thread running on a single core. To remove disk overhead from our measurements and make results more consistent, the entire trace file on disk is read into memory and payloads are pre-extracted from pcap files, although flows are not pre-assembled.

\section{Automaton Construction: Time \& Size}

Figure V-C shows the automaton construction time for each of the BCS sets divided by the number of NFA states. This normalization reduces the variation in construction time due to the underlying complexity of the RegEx set, and allows for easier comparison of the construction methods. Comparing HASIC $_{M}$ and HASIC $_{D}$, we observe that HASIC $_{M}$ is much faster in construction (peaking at 4700 times faster than HASIC $_{D}$ for C7p), and has a more consistent construction time per NFA state than HASIC $D$. Comparing DFA and HASIC $_{D}$, we observe that they take almost the same construction time for each RegEx set. For B217p, which is too complex to be generated by both $\mathrm{HASIC}_{D}$ and DFA, it can be generated by HASIC $_{M}$ in 25.2 seconds.

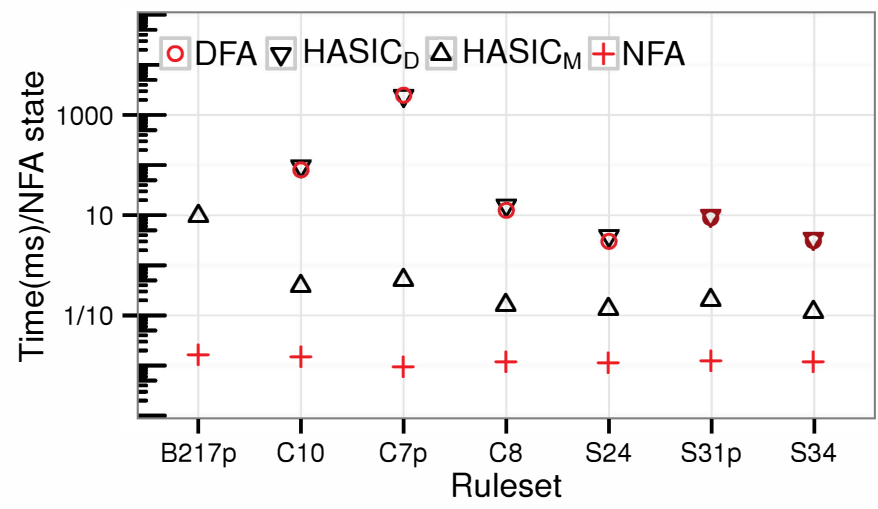

Fig. 6. Construction Time BCS Sets

To evaluate how well $\mathrm{HASIC}_{M}$ construction scales with the complexity of the input, we use the Scale RegEx set. We generated DFA and HFA for the first rule, then the first two rules, then the first three, etc. We stopped generating DFA when the DFA generation time exceeded 2 minutes, while each of the 77 HFAs were generated in under 2.2 seconds. The results in Figure V-C show that $H_{A S I C}$ scales linearly with the number of RegExes while the DFA has exponential construction cost.

Table 8 shows memory image sizes for the various rulesets. The DFA memory images use 4-byte transitions, and the HFA memory images uses 16-byte transitions. Although HFA 


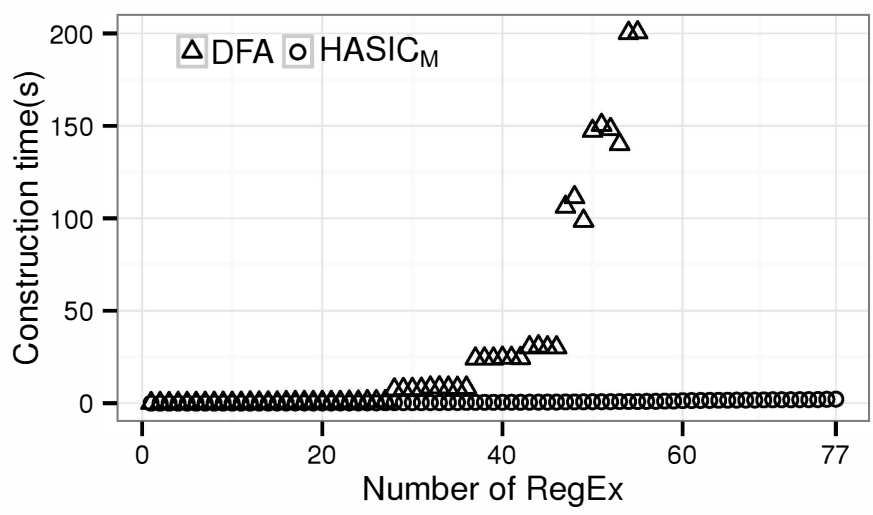

Fig. 7. Construction Time Scale Sequence

has much larger memory size per transition than DFA, for complex RegEx sets, the memory image size of HFA is orders of magnitude smaller than DFA, because HFA has orders of magnitude fewer states. The memory image size of the HFAs constructed by $\mathrm{HASIC}_{M}$ is on average $33 \%$ larger than that of $\mathrm{HASIC}_{D}$.

\begin{tabular}{|r|r|r|r|r|}
\hline Ruleset & NFA & DFA & HASIC $_{D}$ & HASIC $_{M}$ \\
\hline B217p & 0.5 & - & - & 108 \\
\hline C7p & 0.1 & 250 & 4 & 4 \\
\hline C8 & 0.1 & 4 & 0.7 & 0.8 \\
\hline C10 & 0.1 & 20 & 2 & 2 \\
\hline S24 & 0.2 & 10 & 5 & 6 \\
\hline S31p & 0.4 & 41 & 9 & 16 \\
\hline S34 & 0.3 & 13 & 6 & 9 \\
\hline Scale & 0.8 & 608 & 32 & 54 \\
\hline
\end{tabular}

Fig. 8. Memory Image Sizes

\section{Packet Processing Throughput}

Figure V-D shows 6 categories synthetic traces of increasing degree of maliciousness and their impact on the processing throughput of different automata. Note that malicious traces cause RegEx matching performance to drop significantly because they cause the automaton to access a wide variety of its states, which causes the cache hit rate to drop, requiring more accesses to slower main memory. We observe that HFA throughput is 2.9 to 3.6 times, with an average of $3.3 \mathrm{x}$, faster than XFA. XFA performance is estimated from the measurements in [20], which shows that XFA is 75.6/11.6 = 6.5 times slower than DFA. Applying this proportion to our DFA results gives estimated XFA throughput.

For real network traces, the results are similar to random traces, as shown in Figure V-D. Each marker indicates the throughput of a RegEx engine on a single trace. For all automaton types, RegEx sets, and traces, the results were nearly identical to random traces because these traces have a low number of matches on our RegEx sets. The real traces had additional overhead of switching between flow states as packets of different flows arrived and were analyzed, but this did not reduce their performance significantly as compared to the random traces.

Finally, we evaluate the impact of the HFA optimization technique of ordering transitions based on hit probability of

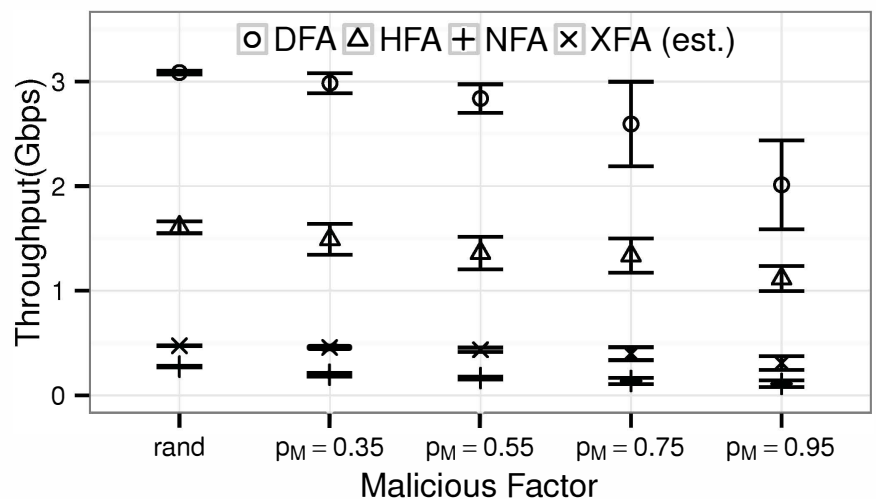

Fig. 9. Throughput Synthetic Traces

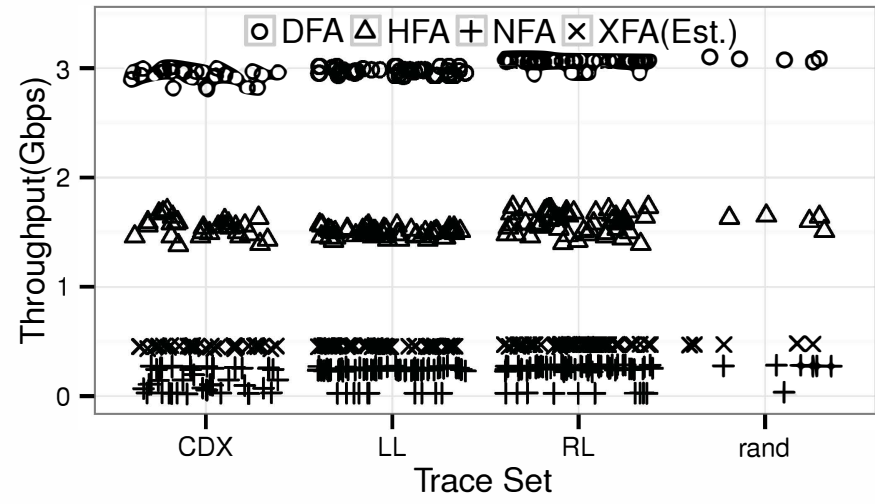

Fig. 10. Throughput Real Traces

each transition. Figure V-D shows the average number of HFA transitions examined for each input character without and with this optimization for synthetic traces generated with $p_{M}$ being 0.35 and 0.75 , respectively. The results show that this optimization technique greatly reduces the average number of HFA transitions examined for each input character and improves packet processing throughput. The optimization is performed using the first $10 \%$ of the trace, and then the number of transitions examined on the rest of the trace is shown. In practice, the average number of HFA transitions examined for each input character with this optimization on a real packet trace will vary depending the similarity between past traffic and current traffic.

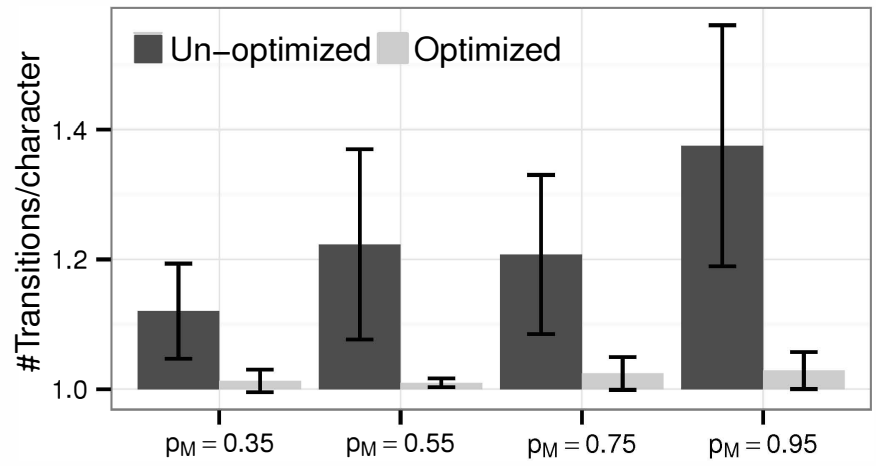

Fig. 11. Transition Order Optimization 
The hardware implementation of HASIC has throughput dependent on two factors. The more important factor, and the one that we have control over, is the memory subsystem it uses. The random-access throughput of the memory subsystem determines the number of transitions per second it can process. The second factor is the number of transitions we need to examine for an input character. Figure V-D shows that we can get the average number of transitions per character down to less than 1.1 after optimization. Table 12 shows the throughput based on an estimate of 1.1 transitions per character and IBM $32 \mathrm{~nm}$ eDRAM technology.

\begin{tabular}{|l|l|l|l|l|}
\hline Memory speed & $\begin{array}{l}\text { \#Read } \\
\text { Ports }\end{array}$ & $\begin{array}{l}\text { Read } \\
\text { Latency }\end{array}$ & $\begin{array}{l}\text { \# HASIC } \\
\text { Engines }\end{array}$ & $\begin{array}{l}\text { Throughput } \\
\text { (Gbps) }\end{array}$ \\
\hline eDRAM @1GHz & 1 & 2 & 1 & 7.3 \\
eDRAM @1GHz & 2 & 2 & 2 & 14.5 \\
eDRAM @1GHz & 4 & 2 & 4 & 29.1 \\
\hline
\end{tabular}

Fig. 12. HASIC hardware implementation throughput

\section{CONCLUSIONS}

As the core problem of many security and networking applications, RegEx matching has received much work; however, it has remained an unsolved problem as it is inherently difficult to achieve high speed with low memory. This work significantly pushes forward the direction of ASIC friendly RegEx matching with high speed and low memory. Using only a few history bits, our algorithms are able to achieve DFAlike matching speed with NFA-like memory. Our algorithms are not only fast in its software implementation but also easy to implement in ASIC due to the simplicity of the RegEx matching process and memory image.

\section{Acknowledgement}

This work is partially supported by the National Science Foundation under Grant Numbers CNS-0845513, CNS-1017588, and CNS-1017598, and the National Natural Science Foundation of China under Grant Number 61272546.

\section{REFERENCES}

[1] Darpa intrusion detection evaluation data set. www.ll.mit.edu/mission/ communications/ist/corpora/ideval/data/1998data.html, 1998.

[2] Us army itoc research $c d x 2009$ trace. http://www.itoc.usma.edu/ research/dataset/index.html, 2009.

[3] M. Bando, N. Artan, and H. Chao. Scalable lookahead regular expression detection system for deep packet inspection. Networking, IEEE/ACM Transactions on, 20(3):699 -714, june 2012.

[4] M. Becchi and P. Crowley. A hybrid finite automaton for practical deep packet inspection. In Proc. of ACM CoNEXT. ACM, 2007.
[5] M. Becchi and P. Crowley. An improved algorithm to accelerate regular expression evaluation. In Proc. ANCS, 2007.

[6] M. Becchi and P. Crowley. Extending finite automata to efficiently match perl-compatible regular expressions. In Proc. CoNEXT, pages $1-12,2008$.

[7] M. Becchi, M. Franklin, and P. Crowley. A workload for evaluating deep packet inspection architectures. In Proc. IEEE IISWC, 2008.

[8] I. Bonesana, M. Paolieri, and M. D. Santambrogio. An adaptable fpgabased system for regular expression matching. In Proc. DATE, pages 1262-1267, 2008.

[9] C. R. Clark and D. E. Schimmel. Efficient reconfigurable logic circuits for matching complex network intrusion detection patterns. In Proc. Field-Programmable Logic and Applications, pages 956-959, 2003.

[10] J. Kořenek and $\mathrm{V}$. Košař. Nfa split architecture for fast regular expression matching. In Proc. ANCS, pages 14:1-14:2, New York, NY, USA, 2010. ACM.

[11] S. Kumar, B. Chandrasekaran, J. Turner, and G. Varghese. Curing regular expressions matching algorithms from insomnia, amnesia, and acalculia. In Proc. ANCS, pages 155-164, 2007.

[12] S. Kumar, S. Dharmapurikar, F. Yu, P. Crowley, and J. Turner. Algorithms to accelerate multiple regular expressions matching for deep packet inspection. In Proc. SIGCOMM, pages 339-350, 2006.

[13] C.-H. Lin, C.-T. Huang, C.-P. Jiang, and S.-C. Chang. Optimization of regular expression pattern matching circuits on fpga. In Proc. DATE, pages 12-17, 2006.

[14] C. Meiners, J. Patel, E. Norige, E. Torng, and A. Liu. Fast regular expression matching using small tcams for network intrusion detection and prevention systems. In Proc. 19th USENIX Security, 2010.

[15] A. Mitra, W. Najjar, and L. Bhuyan. Compiling pcre to fpga for accelerating snort ids. In Proc. ANCS, pages 127-136, New York, NY, USA, 2007. ACM

[16] V. Paxson. Bro: a system for detecting network intruders in real-time. Computer Networks, 31(23-24):2435-2463, 1999.

[17] K. Peng, S. Tang, M. Chen, and Q. Dong. Chain-based dfa deflation for fast and scalable regular expression matching using tcam. In Proc. ANCS, pages 24-35, Washington, DC, USA, 2011. IEEE Computer Society.

[18] M. Roesch. Snort: Lightweight intrusion detection for networks. In Proc. 13th Systems Administration Conference (LISA), USENIX Association, pages 229-238, November 1999.

[19] R. Sidhu and V. K. Prasanna. Fast regular expression matching using fpgas. In Proc. IEEE FCCM, pages 227-238, 2001.

[20] R. Smith, C. Estan, and S. Jha. Xfa: Faster signature matching with extended automata. In Proc. IEEE S\&P, pages 187-201, 2008.

[21] R. Smith, C. Estan, S. Jha, and S. Kong. Deflating the big bang: fast and scalable deep packet inspection with extended finite automata. In Proc. SIGCOMM, pages 207-218, 2008.

[22] L. Yang, R. Karim, V. Ganapathy, and R. Smith. Fast, memory-efficient regular expression matching with NFA-OBDDs. Computer Networks, 55(55):3376-3393, 2011.

[23] Y.-H. E. Yang, W. Jiang, and V. K. Prasanna. Compact architecture for high-throughput regular expression matching on fpga. In Proc. ANCS, pages 30-39, 2008.

[24] F. Yu, Z. Chen, Y. Diao, T. V. Lakshman, and R. H. Katz. Fast and memory-efficient regular expression matching for deep packet inspection. In Proc. ANCS, pages 93-102, 2006. 